\title{
A Geographic Unicast Routing Algorithm using no Location Service
}

\author{
Daniel Silvestre, IEEE Member \\ Inesc-ID \\ Av. Prof. Cavaco e Silva \\ 2744-016 Porto Salvo \\ Email: dsilvestre@tagus.inesc-id.pt
}

\author{
Teresa Vazão, IEEE Senior Member \\ Inesc-ID/IST \\ Av. Prof. Cavaco e Silva \\ 2744-016 Porto Salvo \\ Email: teresa.vazao@ist.utl.pt
}

\begin{abstract}
In this paper, we present a geographic routing algorithm which can adapt to different levels of mobility by changing its parameters according to the network in which it is running. Routing decisions are based on directions and geographical positions of the nodes and there is no need for an external location system. Discoveries are done using unicast messages resulting in few control messages being sent both to discover and to maintain routing information. After a series of tests, we show the algorithm low overhead, high adaptability and robustness as it only relies on the end-points (source and destination nodes) to guarantee a successful transmission.
\end{abstract}

Keywords-ad-hoc; geographic; routing; location-based;

\section{INTRODUCTION}

Mobile ad hoc networks (MANETs) are formed independently by nodes that temporarily want to communicate with no central unit controlling the network. The absence of a predetermined infrastructure represents some advantage to the deployment of MANET as no investment on infrastructures is required. As the routing task is distributed over devices with less resources (such as weaker antennae, slower processing units and less memory) there are less costs and a more scalable solution.

Nevertheless, such unstructured networks present additional problems and create more challenges to the protocols used. The use of radio transmission presents signal propagation problems such as attenuation, fading, shading or multipath propagation [1]. This leads to a greater Bit Error Rate (BER) on the channel and mobility can break paths and lead to successive retransmissions. Moreover, the use of a shared wireless medium leads to congestion and thus, less performance is achieved when the number of hops increases.

Different types of routing protocols tackled the problem in distinct ways. In initial protocols, nodes retransmit control messages to all the other nodes (broadcast) which is expensive in terms of resources and scalability. Evolutions such as Geographic protocols use location to perform better decisions, although there is the need for an external location system in most cases. The use of unicast (i.e., control messages are not retransmitted to all the nodes in the network) is essential in order to maximise the routing performance.

We target MANETs with high mobility and our main objectives are to (i) lower overhead, (ii) decrease latency and jitter, (iii) achieve a good delivery ratio. Therefore, we shall investigate those metrics during our evaluation.

This paper presents a new routing algorithm called SWAN (Simple Wide-deploy Algorithm for ad hoc Networks), whose main contributions are:

- Low overhead: achieved through a route discovery based on unicast communication instead of broadcast;

- Near optimal routes: a message is forwarded along an optimal path in distance at each given time;

- High delivery ratio: nodes act as proxy for data messages when there is no possible next hop;

- Absence of a route repair process: routing decisions are made on a per-packet basis using solely on the sender and receiver coordinates, avoiding controlled floods;

- Self-adaptive: the algorithm adapts its parameters, preventing errors from misconfiguration and potentially increasing its performance in highly mobile networks;

- Capable of sending messages in network with voids or fragmented using the angle correction and the proxy state.

Contact the author D. Silvestre through dsilvestredisr.ist.utl.pt for a free unformatted copy of this article or go to http://ieeexplore.ieee.org/abstract/document/5598194/

for the final formatted version.

\section{REFERENCES}

[1] J. Schiller, Mobile Communication, 2nd Edition, AddisonWesley, August 2003.

[2] David B. Johnson and David A. Maltz, Dynamic Source Routing in Ad Hoc Wireless Networks, The International Series in Engineering and Computer Science, Vol. 353, pp. 153-181, Springer US, 1996.

[3] C. Perkins and P. Bhagwat, Highly Dynamic DestinationSequenced Distance-Vector Routing (DSDV) for Mobile Computers, 1994.

[4] C. Perkins, E. Belding-Royer and S. Das Ad hoc On-Demand Distance Vector (AODV) Routing, RFC 3561, IETF, July 2003.

[5] T. Clausen and P. Jacquet Optimized Link State Routing (OLSR) Protocol, RFC 3626, IETF, October 2003. 
[6] M. Dorigo, V. Maniezzo, and A. Colorni, Ant system: optimization by a colony of cooperating agents, IEEE Transactions on Systems, Man, and Cybernetics, Part B: Cybernetics, vol.26, no.1, pp.29-41, Feb 1996.

[7] A. Vahdat and D. Becker, Epidemic routing for partiallyconnected ad hoc networks, Technical report, 2000.

[8] M. Chen, V. Leung, S. Mao and Y. Yuan, Directional geographical routing for real-time video communications in wireless sensor networks, Computer Communications, Vol. 30, N. 17, 2007.

[9] B. Karp and H. Kung, GPSR: greedy perimeter stateless routing for wireless networks, ACM MobiCom '00: Proceedings of the 6th annual international conference on Mobile computing and networking, pp. 243-254,New York, NY, USA, 2000.

[10] N. Chaki and R. Chaki, ORRP: Optimal Reactive Routing Protocol for Mobile Ad-Hoc Networks, CISIM '07 6th International Conference on Computer Information Systems and Industrial Management Applications, pp.185-190, 28-30 June, 2007.

[11] IEEE Computer Society LAN MAN Standards Committee. Wireless LAN Medim Access Control (MAC) and Physical Layer (PHY) Specifications, The Institute of Electrical and Electronics Engineers, New York, New York, 1997.

[12] M. Mauve, J. Widmer and H. Hartenstein, A Survey on Position-Based Routing in Mobile Ad-Hoc Networks, IEEE Network, pp 30-39, vol. 15, 2001.

[13] D. Chen and P. Varshney, A survey of void handling techniques for geographic routing in wireless networks, IEEE Communications Surveys and Tutorials, 2007. 